\section{(6) OPEN ACCESS}

\title{
A basal gradient of Wnt and stem-cell number influences regional tumour distribution in human and mouse intestinal tracts
}

\author{
Simon J Leedham, ${ }^{1,2}$ Pedro Rodenas-Cuadrado, ${ }^{1}$ Kimberley Howarth, ${ }^{1}$ \\ Annabelle Lewis, ${ }^{1}$ Sreelakshmi Mallappa, ${ }^{3}$ Stefania Segditsas, ${ }^{1}$ Hayley Davis, ${ }^{1}$ \\ Rosemary Jeffery, ${ }^{4}$ Manuel Rodriguez-Justo, ${ }^{5}$ Satish Keshav, ${ }^{2}$ \\ Simon P L Travis, ${ }^{2}$ Trevor A Graham, ${ }^{4}$ James East, ${ }^{2}$ Susan Clark, ${ }^{3}$ \\ Ian P M Tomlinson ${ }^{1}$
}

- Additional materials are published online only. To view these files please visit the journal online (http://dx.doi.org/ 10.1136/gutjnl-2011-301601).

${ }^{1}$ Molecular and Population Genetics, Wellcome Trust Centre for Human Genetics, University of Oxford, UK ${ }^{2}$ Translational Gastroenterology Unit, John Radcliffe Hospital, Oxford, UK

${ }^{3}$ Polyposis Registry, St Mark's Hospital, Harrow, UK ${ }^{4}$ Histopathology Laboratory, London Research Institute, London, UK

${ }^{5}$ Histopathology department, University College London Hospital, London, UK

\section{Correspondence to} Dr Simon Leedham, Molecular and Population Genetics, Wellcome Trust Centre for Human Genetics, University of Oxford, Roosevelt Drive, OX3 7BN, UK;

simon.leedham@cancer.org.uk

\section{Final note During the} preparation of this manuscript Albuquerque et al published a paper screening human colorectal tumours for mutations in Wnt related genes and have independently proposed a similar mechanism to that suggested by us for the apparent non-random distribution of human colorectal tumours. $^{27}$

Accepted 15 December 2011 Published Online First 27 January 2012

\section{ABSTRACT}

Objective Wnt signalling is critical for normal intestinal development and homeostasis. Wnt dysregulation occurs in almost all human and murine intestinal tumours and an optimal but not excessive level of Wnt activation is considered favourable for tumourigenesis. The authors assessed effects of pan-intestinal Wnt activation on tissue homeostasis, taking into account underlying physiological Wnt activity and stem-cell number in each region of the bowel.

Design The authors generated mice that expressed temporally controlled, stabilised $\beta$-catenin along the crypt-villus axis throughout the intestines. Physiological Wnt target gene activity was assessed in different regions of normal mouse and human tissue. Human intestinal tumour mutation spectra were analysed.

Results In the mouse, $\beta$-catenin stabilisation resulted in a graduated neoplastic response, ranging from dysplastic transformation of the entire epithelium in the proximal small bowel to slightly enlarged crypts of non-dysplastic morphology in the colorectum. In contrast, stem and proliferating cell numbers were increased in all intestinal regions. In the normal mouse and human intestines, stem-cell and Wnt gradients were non-identical, but higher in the small bowel than large bowel in both species. There was also variation in the expression of some Wnt modulators. Human tumour analysis confirmed that different APC mutation spectra are selected in different regions of the bowel.

Conclusions There are variable gradients in stem-cell number, physiological Wnt activity and response to pathologically increased Wnt signalling along the cryptvillus axis and throughout the length of the intestinal tract. The authors propose that this variation influences regional mutation spectra, tumour susceptibility and lesion distribution in mice and humans.

\section{INTRODUCTION}

Wnt signalling plays a critical role in the development and homeostasis of the intestinal epithelium. Among other functions, the Wnt pathway is centrally involved in the maintenance of the stemcell phenotype, control of epithelial cell localisation along the crypt-villus axis, secretory lineage development and the maturation of Paneth cells

\section{Significance of this study}

What is already known about this subject?

- Increased Wnt/ $\beta$-catenin signalling underlies the initiation and progression of gastrointestinal tumours.

- An optimal but not excessive level of Wnt activation is considered favourable for tumourigenesis: the 'just-right' theory.

- There is marked variation in the distribution of murine and human intestinal tumours with a preponderance of small intestinal lesions in the mouse and colorectal tumours in the humans.

- Human tumour mutation spectra vary throughout the intestinal tract. For example, microsatellite unstable tumours are predominantly found on the right side of the colon.

\section{What are the new findings?}

- In a mouse model, stabilisation of $\beta$-catenin resulted in graduated neoplastic response down the length of the intestinal tract, with a marked increase in the number of stem and proliferating cells throughout.

- This graduated response mirrored underlying physiological gradients of stem cells, Wnt target gene activity and expression of some Wnt modulators throughout the intestines of normal mice.

- Similar but non-identical physiological gradients were found along normal human intestinal tracts and we have confirmed that human tumour $A P C$ mutation spectra are selected in different regions of the bowel.

- We propose that variation in stem-cell number and physiological Wnt activity influences regional mutation spectra, tumour susceptibility and lesion distribution.

(reviewed in Scoville et $a l^{1}$ ). The reliance of the intestinal epithelium on physiological Wnt signalling was demonstrated by Kuhnert et al who demonstrated catastrophic loss of adult mouse 


\section{Significance of this study}

\section{How might it impact on clinical practice in the near} future?

- This work improves our understanding of the mechanisms behind the non-random tumour distribution in human intestinal cancer.

- Targeted epithelial and mesenchymal manipulation of Wnt in colorectal cancers may prove to be an effective therapeutic strategy.

intestinal epithelial proliferation and crypt structure $7-10$ days after adenoviral expression of the Wnt antagonist Dickkopf-1.

Wnt signalling is dysregulated in almost all intestinal neoplasia in the mouse and humans (reviewed by Radtke et $\mathrm{al}^{3}$ ). The adenomatous polyposis coli (APC) protein is a component of the canonical Wnt signalling pathway and is responsible for the regulation of the transcription factor $\beta$-catenin. Truncation or loss of APC disrupts the $\beta$-catenin degradation complex, increasing nuclear translocation of this transcription factor and causing increased expression of Wnt target genes (reviewed by Sieber et $a l^{4}$ ). Germline APC mutations are responsible for familial adenomatous polyposis (FAP), an autosomal dominant condition characterised by the formation of 100's to 1000's of colonic polyps. Most patients also develop adenomas of the duodenum, with many developing neoplastic gastric lesions (adenomas or fundic gland polyps) and the severity of colonic, gastric and duodenal polyposis varies within and among families. Somatic mutations in $A P C$ are also found in the majority of sporadic colonic adenomas ${ }^{5}$ and carcinomas. ${ }^{6}$

In human colorectal tumours, biallelic APC mutations occur non-randomly with respect to one another. ${ }^{7}$ Mutations are selected on their combined ability to produce an optimal, but not excessive, level of $\beta$-catenin activation in the tumour cell, a model subsequently dubbed the 'just-right' hypothesis. ${ }^{8}$ The majority of somatic APC mutations occur between codons 1250 and 1514 , termed the mutation cluster region. ${ }^{69}$ This region of the gene encodes the first three of seven 20 -amino acid $\beta$-catenin binding repeats (20AARs). Mutations in the mutation cluster region affect the numbers of 20AARs retained by the encoded protein and so alter its ability to bind and degrade $\beta$-catenin. In general, retention of a greater number of repeats results in more modest Wnt perturbation in the resultant polyp.

The number of 20AARs retained within a tumour varies according to the region of the intestinal tract in which the lesion is located. In FAP, germline mutations around codon 1300 are associated with copy-neutral loss of heterozygosity (LOH) and a very severe colonic phenotype. This mutation combination results in retention of one 20AAR per allele and is optimally selected in the rectal lesions of germline 1309 patients. ${ }^{10}$ Severe duodenal polyposis is associated with germline mutations after codon 1400 , and somatic mutations that result in a cumulative total of four or more 20AARs in each upper gastrointestinal (GI) lesion are strongly selected. ${ }^{11}$ Similar variability in mutation distribution is seen in sporadic colorectal tumours, with rightsided tumours retaining significantly more 20AARs than leftsided lesions. ${ }^{12}$

Analysis of the first hit/second hit relationship in human tissue is observational, based upon the site of APC mutations in established lesions. To further analyse the optimal level of Wnt signalling for tumourigenesis, Apc-mutant mice with different numbers of retained 20AARs have been generated. We recently undertook a detailed molecular and phenotypic analysis of two Apc-mutant mice on the C57BL/6 background, $A p c^{\operatorname{Min}(R 850 X)}$ and $A p c^{1322 T 13}{ }^{14}$ In tumours from both these animals the 'second hit' was LOH by mitotic recombination, resulting in cumulative 20AAR totals of 0 and 2, respectively. Polyp distribution was markedly different in these two strains: Apc ${ }^{1322 T}$ animals developed a heavy tumour burden in the proximal small bowel (SB1) (segments 1 and 2) whereas $A p c^{M i n}$ mice had relatively more polyps in the distal small bowel (SB3) (segments 2 and 3).

Although there are differences between mice and humans, such as intestinal polyp location, the collated data show that an optimal level of Wnt signalling for GI tumourigenesis exists and that this level is submaximal. The evidence also demonstrates that cells of all types in the normal intestine require an optimal Wnt dosage, lower than in tumourigenesis, at different stages of their progression from stem cell-through progenitor, transit amplifying and differentiated phenotypes - to shedding into the gut lumen. We wished to assess how high-efficiency activation of Wnt signalling throughout the intestines would affect cell and tissue homeostasis, taking into account the underlying level of physiological Wnt signalling in each region of the bowel. We chose to study a transgenic mouse that expressed a temporally controlled, stabilised $\beta$-catenin protein. This mouse was preferred to Apc-mutant animals because $\beta$-catenin purely drives canonical Wnt signalling, preventing any hypothetical variation caused by non-LOH second hits at $A p c$, and avoiding the effects of losing non-Wnt functions of Apc. ${ }^{15}{ }^{16}$ Here, we report the findings from this mouse and from our subsequent assessment of Wnt signalling in normal mouse and human intestines.

\section{METHODS}

\section{Transgenic stabilised $\beta$-catenin mouse}

Physiological degradation of the $\beta$-catenin protein occurs when GSK3 $\beta$-mediated phosphorylation of serine/threonine residues signals for rapid ubiquitin-dependent proteosomic degradation. These serine/threonine residues are coded for by exon 3 of the gene. Harada et al generated a transgenic mouse with exon 3 flanked by loxP sites $\left(C_{t n n b} 1^{\text {lox }(e x 3) /+}\right) .{ }^{17}$ Recombination with an intestinal driven Cre allowed transgenic in-frame deletion of the entire exon resulting in the expression of degradation resistant $\beta$-catenin. ${ }^{17}$ Subsequently, El Marjou et al ${ }^{18}$ demonstrated that a 9-kb regulatory region of the villin gene targeted stable and homogeneous expression of transgenes along the crypt-villus axis in the small and large intestines. We used this inducible Villin-cre system (Villin-Cre ${ }^{E R T 2}$ ) crossed with a Ctnnb1 10x(ex3)/+ mouse on a C57BL/6 genetic background (gift of Mark Taketo) to allow temporal control over pan-intestinal epithelial $\beta$-catenin stabilisation in adult animals. Following Cre-mediated recombination, we denoted the mice as Ctnnb $1^{\text {tex3 }}$. Genotyping and tissue collection are described in the online supplementary methods.

\section{Assessment of colonic Cre-mediated recombination}

In order to ensure that Cre recombination occurred uniformly and efficiently in the large intestine of the transgenic animals, we measured the amount of residual $\beta$-catenin exon 3 DNA using a standard absolute quantification method (online supplementary methods). Colonic epithelial tissue was laser dissected to minimise contamination from underlying normal stromal tissue and leucocytes. Normalised values for an epithelial cell population were expected to be close to 1 for control animals and 0.5 for heterozygous mice. 
Laser capture microdissection and RNA extraction for stem-cell marker qRT-PCR

Six $5 \mu \mathrm{m}$ serial sections were cut from the wild-type and transgenic colonic tissue blocks used for in situ hybridisation (ISH) and individual crypts were microdissected as previously described. ${ }^{19}$ RNA from individual crypts was extracted using Arcturus Paradise FFPE RNA isolation kit (Molecular Devices, Sunnyvale, California, USA) and underwent reverse transcription, amplification and quantitative reverse transcriptase PCR (qRT-PCR).

\section{Immunohistochemistry}

Immunohistochemistry for differentiated cell types, cell proliferation, apoptosis and $\beta$-catenin expression was completed using standard techniques (online supplementary methods and supplementary table 1).

Cell counting and statistical power

Crypt size, cell proliferation, stem-cell number, apoptotic cells and lineage marker expression were compared between a minimum of 100 crypts or crypt-villus units from each intestinal region, from a total of four Ctnnb1 ${ }^{\Delta \mathrm{ex} 3}$ and wild-type littermates. Cells were counted by three different investigators (SL,
PRC, AL), blinded to the mouse genotype. The power to discern differences in cellularity between two intestinal compartments depends upon the magnitude of the difference between the compartments. By contrasting a minimum of 100 crypts from mutant animals to 100 crypts from wild-type animals we were able to detect effect sizes of approximately 0.7 with $99 \%$ power (assuming a t test was appropriate to compare between groups of $n=100$, and requiring a significance level of 0.01 ).

\section{In situ hybridisation}

Mouse model lineage tracing experiments have demonstrated that crypt based columnar cells specifically expressing Leucine rich repeat containing G-protein coupled receptor 5 (Lgr5) are capable of self-replication and multilineage differentiation in the intestine and colon. ${ }^{20}$ mRNA ISH for Lgr 5 as a putative stem-cell marker was carried out on human duodenal, ileal and colonic biopsies from seven different patients and wild-type and transgenic mouse gut tissue as described in the online supplementary methods.

\section{Normal mouse and human whole mount crypt analysis}

Freshly dissected circles of wild-type mouse tissue from the SB1, mid-small bowel (SB2) and SB3, caecum and rectum were obtained using a $2 \mathrm{~mm}$ diameter punch biopsy tool. From the
Figure 1 Stabilised $\beta$-catenin mouse dysplastic change in the small intestine parallels expansion of proliferating cells with abnormal $\beta$-catenin expression. In all intestinal regions there was expansion of the mid-crypt zone. In the proximal small bowel (SB1) this was so pronounced that it destroyed the cryptvillus architecture (Ai). The expanded mid-crypt cell population was dysplastic, actively proliferating (Ki-67 + immunostaining) (Aiii) and had abnormal expression of $\beta$-catenin (heavy nuclear or cytoplasmic) (Aii). Distal small bowel (SB3) to mid-small bowel (SB2) there was some preservation of normal cell morphology at the crypt bases and villi tips with Ki67 negative cells ( $B, C$ iii) and membranous $\beta$-catenin stain ( $B, C i i)$. The entire colon was morphologically non-dysplastic with membranous $\beta$ catenin staining (Dii); however, there was an expansion and upward shift of the Ki-67+ cells in comparison with wild-type controls (Diii). Expansion of the stem-cell zone using $\mathrm{S}^{35}$-UTP labelled in situ hybridisation for Lgr5 (A-Eiv). There was expansion in the number of Lgr5 expressing cells in all intestinal regions. In the Ctnnb $1^{\Delta \mathrm{ex} 3}$ mouse colon the Lgr5+ cells were restricted to crypt bases but filled the bottom third of the phenotypically nondysplastic crypt (Div), a considerable expansion of the stem-cell compartment in comparison with wildtype mouse colon (Eiv). ii. $\beta$-catenin

immunostain

iii. Ki-67 immunostain

iv. Lgr5 ISH dark field

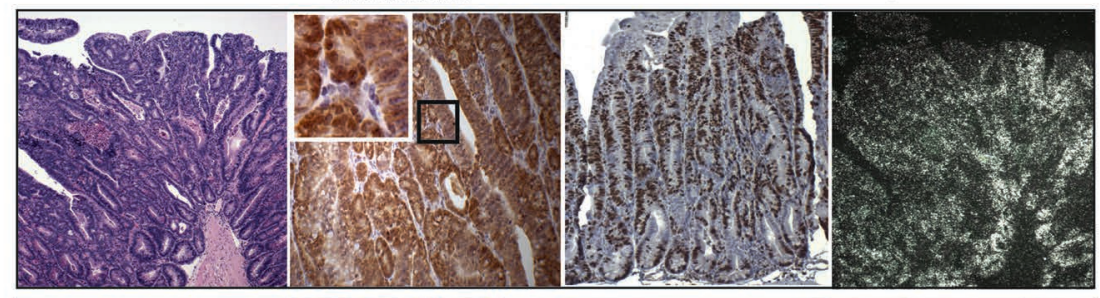

B

SB2

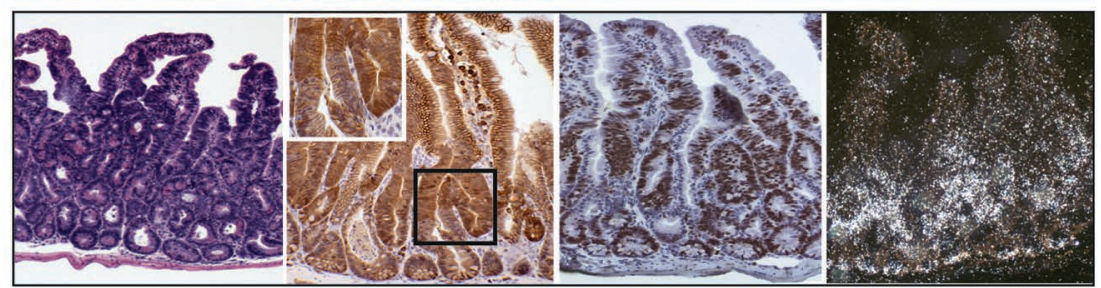

C

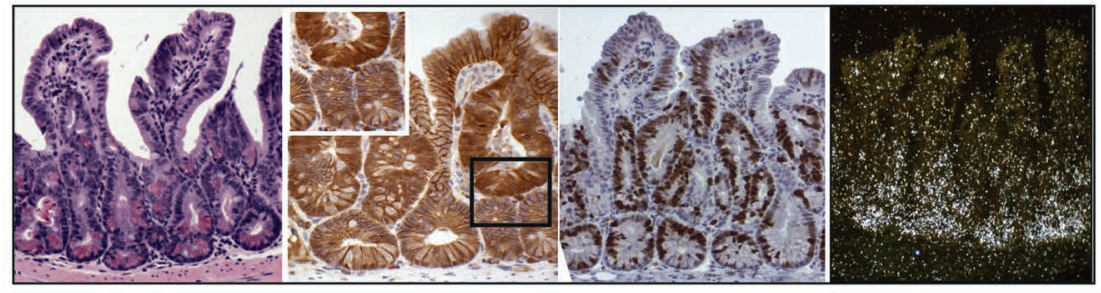

Colon
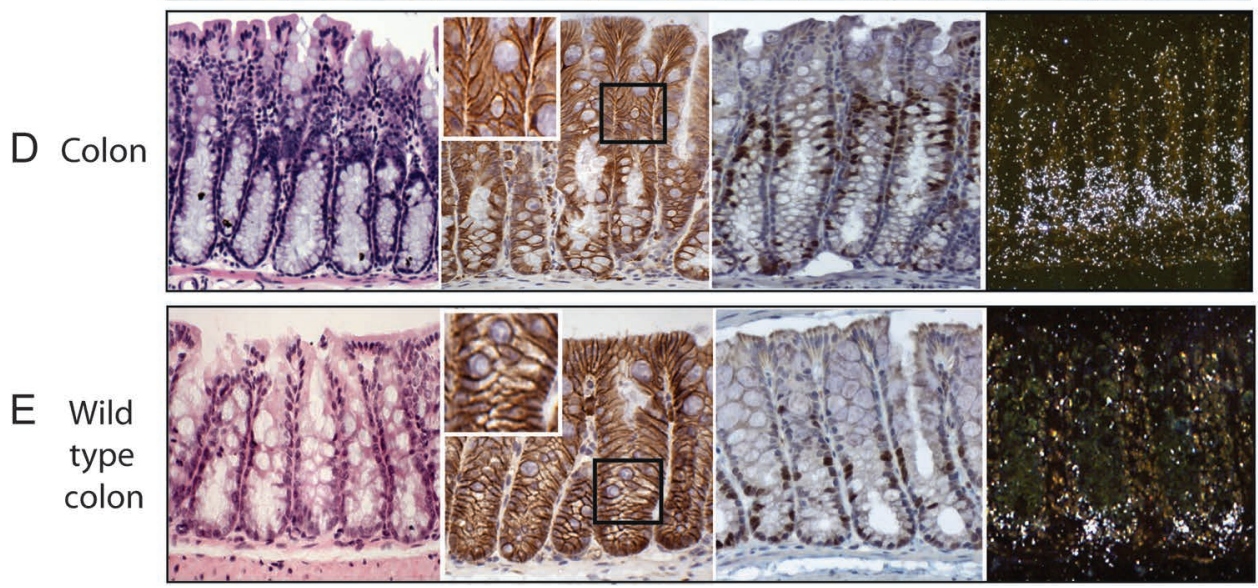
human, biopsies of the duodenum, ileum, caecum and rectum were taken from upper and lower endoscopies performed on the same patient. Individual crypts were isolated by whole mount extraction and underwent RNA extraction and qRT-PCR for analysis of Wnt target gene expression (online supplementary methods). The remaining mesenchymal tissue after epithelial denudement was also isolated and processed.

\section{Archival human sporadic and FAP associated polyps and tumours}

Tissue collection

Upper GI biopsies and colectomy specimens from the same FAP patients and sporadic colorectal tumours with a clearly defined colonic location were obtained from pathology archives. Tissue sectioning, DNA extraction, APC sequencing and $5 q \mathrm{LOH}$ analysis were completed as previously described ${ }^{19}$ (online supplementary methods).

Public mutation databases

The APC mutation status of additional sporadic colorectal tumours or cell lines was collected from the Catologue of Somatic Mutations in Cancer (COSMIC v53 http://www.sanger.ac.uk/ genetics/CGP/cosmic/) (online supplementary methods).

\section{RESULTS}

Pan-intestinal $\beta$-catenin stabilisation causes florid dysplasia in the small intestine but not in the colorectum

Four weeks after Cre induction, Ctnnb1 $1^{\text {Dex3 }}$ animals developed dysplastic changes in the GI tract. The proximal part of the small bowel was grossly thickened with a rubbery texture, but there were no discrete polyps produced either here or in any other part of the intestines. In the duodenum (SB1), microscopic analysis showed that the epithelium was carpeted by high-grade dysplastic cells, with complete destruction of crypt-villus architecture. The morphological appearance gradually improved distally. In the jejunum (SB2), high-grade dysplasia was only ubiquitous at the mid-crypt level. The crypts were greatly enlarged, but there was some preservation of architecture and normal cell morphology, especially at the tips of the villi and bases of the crypts. In the distal ileum (SB3), crypts were enlarged, with a variable mixture of mildly dysplastic and morphologically normal cells extending onto the villi (figure 1). The colon of the Ctnnb1 $1^{\text {ex } 3}$ animals was macroscopically normal and microscopically non-dysplastic, but closer examination showed abnormalities. The colorectal crypts of Ctnnb1 $1^{\text {Dex3 }}$ animals were larger than those of their Ctnnb1-wildtype litter-mates, had significantly more cells $(p<0.0001$,

Table 1 Morphological and immunohistochemical findings from different intestinal regions of wild-type and $\operatorname{Ctnnb} 1^{\Delta e \times 3 /+}$ mice

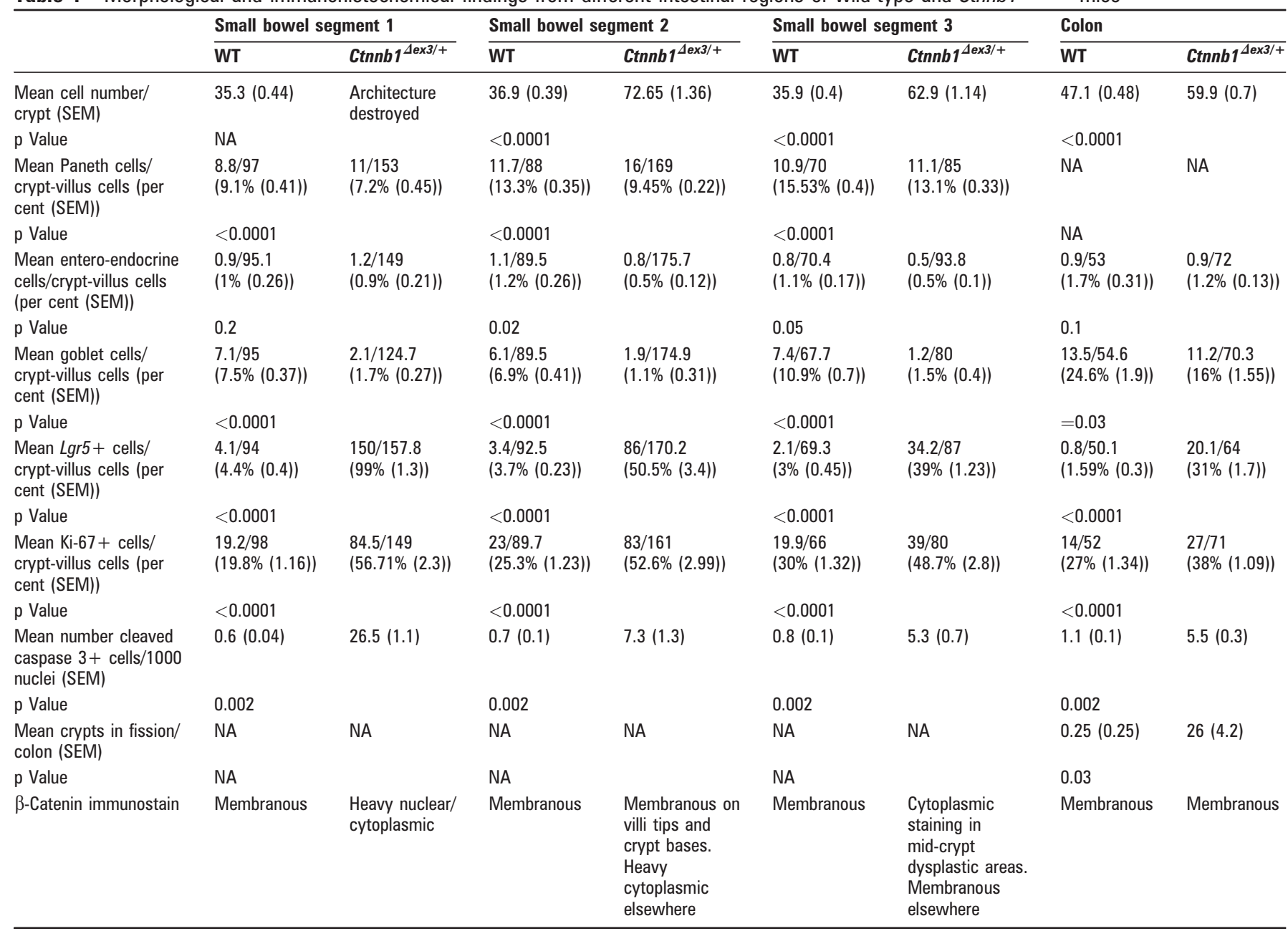

The table shows the mean number and percentage of cells per tissue unit on well-orientated cross-sections. A minimum of 50 crypts/crypt-villus units were counted from four different mutan mice. The number of crypts in obvious fission were counted in entire colon rolls from four wild-type and four mutant mice. $\mathrm{p}$ Values were calculated using Mann-Whitney $\mathrm{U}$ test (Prism, Graphpad Software, La Jolla, California, USA).

WT, wild-type. 
Mann-Whitney $U$ test) and had a higher prevalence of fission ( $\mathrm{p}=0.03$, Mann-Whitney U test) (table 1 ).

All differentiated cell types were present in dysplastic tissue (table 1). Although there was an absolute increase in small intestinal Paneth cell numbers in transgenic tissue, the increase in crypt size led to transgenic crypts having proportionally fewer of these cells than their wild-type counterparts $(p<0.0001$, Mann-Whitney U test). Paneth cells were also displaced from the base in mutant crypts (online supplementary figure 1).

Immunohistochemistry showed $\beta$-catenin expression in $C_{\text {tnnb }}{ }^{\text {ex } 3}$ mice to parallel dysplasia throughout the intestine. In SB1, $\beta$-catenin was predominantly heavily nuclear with some regions of cytoplasmic staining. In SB2 and SB3, expression was cytoplasmic in the dysplastic cells in the mid-crypt zone and predominantly membranous otherwise, including at the preserved villus tips in the SB2 to SB3. Membranous $\beta$ catenin staining was seen in all morphologically non-dysplastic epithelial cells, including the entire large bowel of Ctnnb1 $1^{\Delta e \times 3}$ mice and throughout the intestines of control animals (table 1, figure 1).

Cell proliferation marker expression in the $C_{n n n b 1}{ }^{\Delta e x 3}$ mice correlated strongly with dysplasia and $\beta$-catenin expression. Where crypt-villus architecture was maintained, the proliferative zone filled the dysplastic mid-crypt region. It was significantly enlarged ( $p<0.0001$, Mann Whitney test) and shifted towards the tops of the crypts in comparison with control mice (table 1, figure 1). Apoptosis, as determined by cleaved caspase 3 immunohistochemistry, was increased in all dysplastic tissue $(p=0.002)$ (online supplementary figure 1).

In order to show that the absence of colorectal neoplasia in the colon of transgenic mice was not simply the result of lack of Cre expression and/or recombination in the large intestine, we measured the amount of residual wild-type $\beta$-catenin in laserdissected colonic epithelium from $\mathrm{Ctnnb}_{1}{ }^{\operatorname{ex} 3}$ mice compared with their wild-type litter-mates. The mean dosage of wild-type $\beta$-catenin was 0.49 (SEM 0.02) in $C_{\text {tnnb }}{ }^{\text {dex3 }}$ mice relative to the wild-type animals (mean 1.1 (SEM 0.03)), showing very high efficiency recombination and demonstrating that the nondysplastic colonic phenotype was not simply a consequence of variable intestinal Cre activity.

\section{Stem-cell marker expression in $\mathbf{C t n n b} 1^{\Delta \text { ex } 3}$ mice}

We next assessed the expression of established stem-cell markers in the Ctnnb $1^{\text {ex } 3}$ mice compared with wild-type animals. ISH demonstrated a huge expansion in the Lgr5expressing cell population throughout the small intestine. The most pronounced changes were in SB1, where the entire epithelium was Lgr5+. In SB2 and SB3, Lgr5+ cells were predominantly basal, as in normal tissue, although the stemcell zone was expanded, extending into the mid-crypt region and overlapping with the mid-crypt proliferative/dysplastic zone (figure 1).
Figure 2 Stem-cell numbers vary through the intestinal tract. (A) Mouse in situ hybridisation (ISH). H3-UTP labelled ISH for Lgr5 on well-orientated crypts from a wild-type mouse: (i) proximal small bowel (SB1); (ii) midsmall bowel (SB2); (iii) distal small bowel (SB3); and (iv) colon. Individual cells containing $\geq 4$ silver granules were counted and are highlighted in each picture. (B) Mouse Lgr5+ cell counts. Mean (SEM) Lgr5+ cell counts are shown. Lgr5 + cells are maximal in the SB1 with a slightly reducing number through to the ileum (SB3). A profound drop in $L g r 5+$ cells is seen in the colon $(p<0.001$, analysis of variance). Cell numbers were counted in 100 crypts from each region from three different wild-type mice. (C) Human ISH. $\mathrm{S}^{35}$-UTP labelled ISH for LGR5 on human endoscopic biopsy specimens from: (i) duodenum (ii) ileum and (iii) colon of the same patient. LGR5 expression was restricted to the crypt bases and was maximal in the ileum, with only single LGR5 + cells seen in colonic crypts. ISH was carried out on biopsy sections from 7 different patients undergoing normal upper and lower endoscopies.
A Mouse

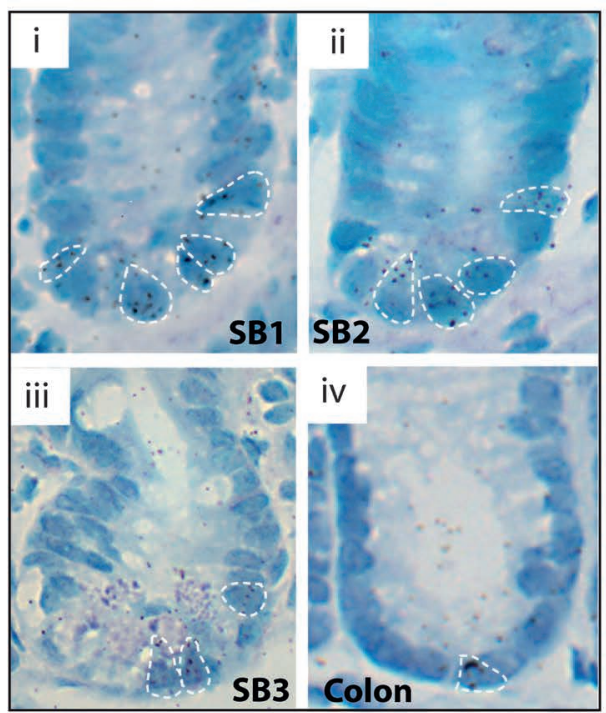

\section{B Cell count}

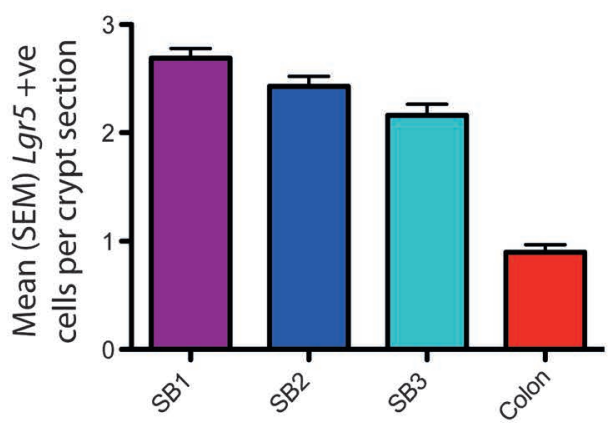

Intestinal segment

\section{Human}

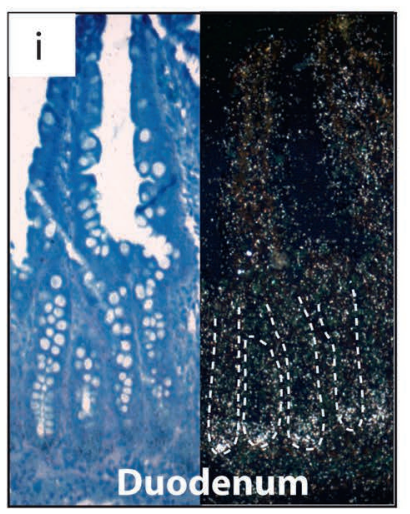

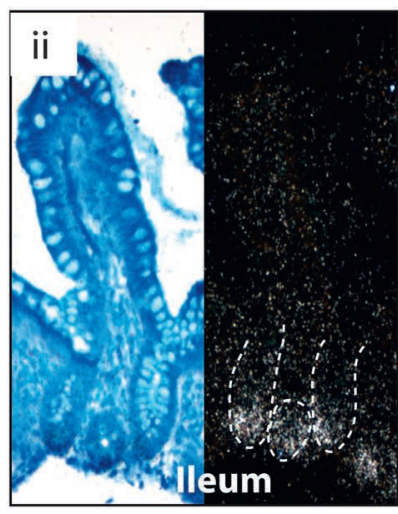

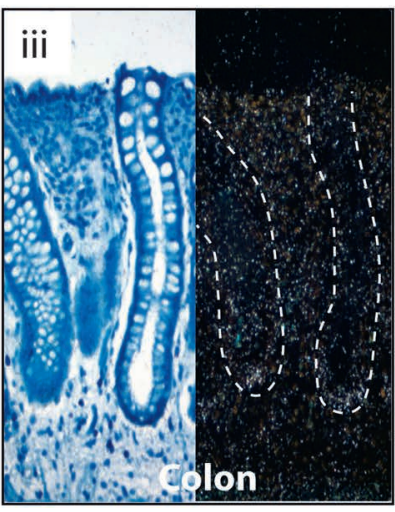


In the large bowel, despite normal morphology, Lgr5+ cells filled the bottom third of the crypt, representing a considerable expansion of the putative stem-cell population in comparison with wild-type controls (figure 1D,E). qRT-PCR on a total of 45 individual laser-microdissected crypts confirmed higher expres- sion of Lgr5 in the large bowel of $\mathrm{Ctnnb}^{\mathrm{Aex3}}$ animals, with a mean 9.2-fold increase (SEM 1.3; $\mathrm{p}=0.002$, t test) compared with wild-type litter-mates. Another colonic stem-cell marker, Ascl2, was also more highly expressed in the mutant animals (mean increase 9.3-fold, SEM 2.3; $\mathrm{p}=0.02$, t test). i. Transit amplifying cells A Mouse

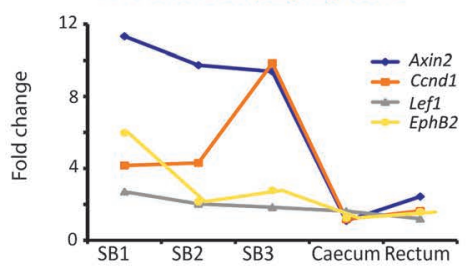

ii. Paneth cells

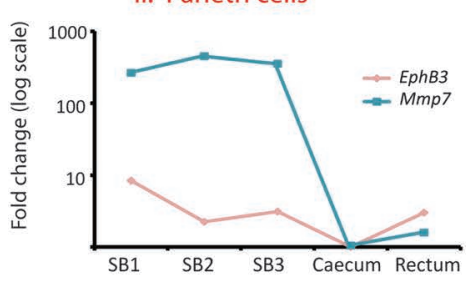

iii. Stem-cell zone

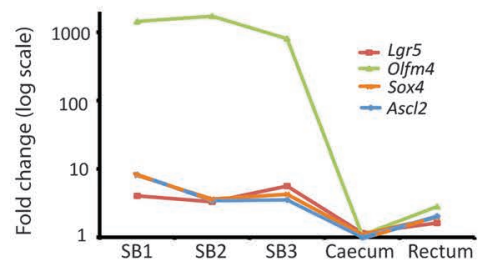

iv. Wnt modulators
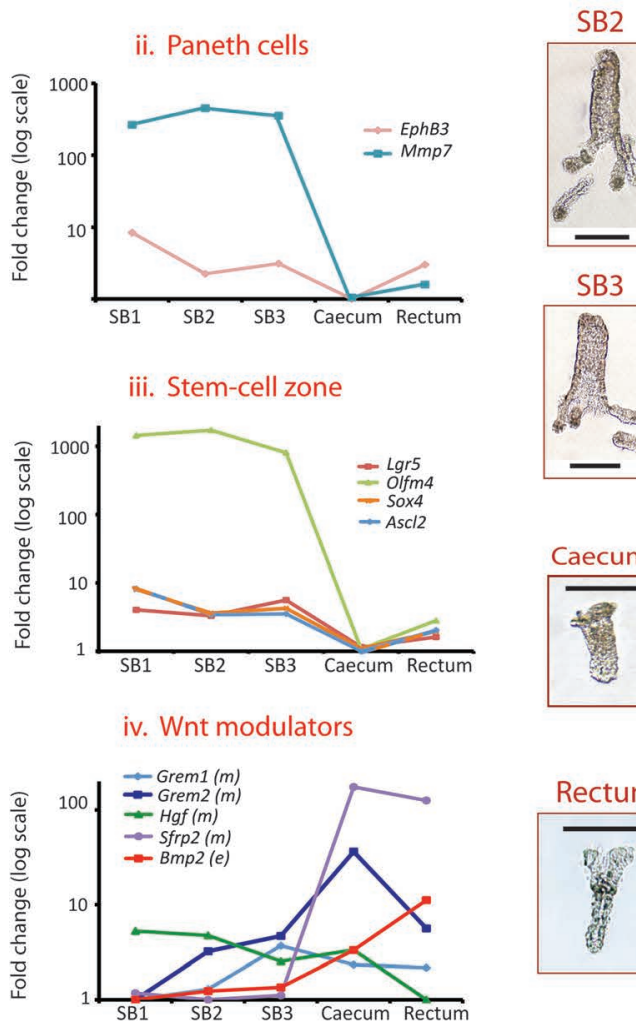

B Human
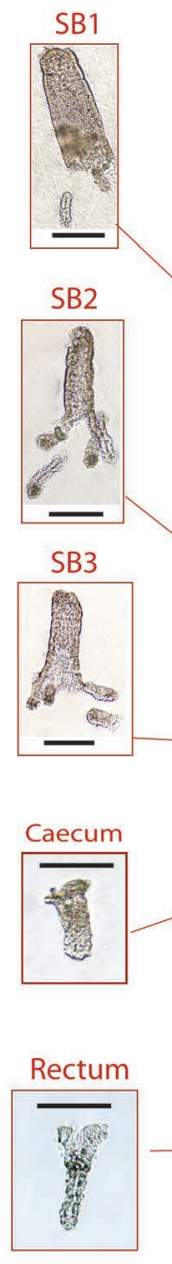

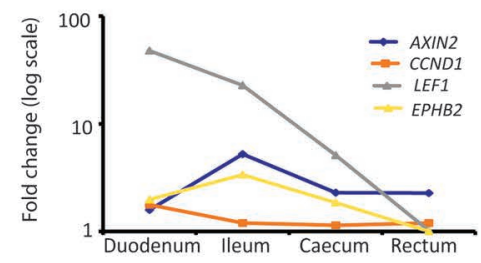

ii. Paneth cells

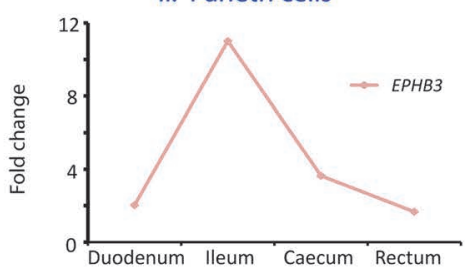

iii. Stem-cell zone

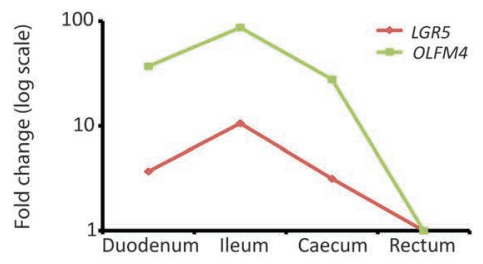

iv. Wnt modulators

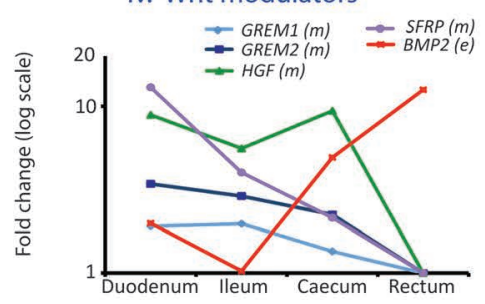

Figure 3 Individual crypt Wnt target gene expression varies longitudinally throughout the normal mouse and human intestinal tract. Thumbnail pictures show whole mount intestinal crypts and villi from the different regions of normal mouse and human intestine after EDTA extraction. Mouse villi are pictured purely to provide scale; in fact, only individual crypts were aspirated and used in analysis. Mesenchymal tissue was isolated after complete epithelial denudement. Scale bar $100 \mu \mathrm{m}$. Five crypts were dissected from each intestinal region, analysed individually and used to calculate a mean $\Delta \mathrm{Ct}$, which was then averaged across three wild-type mice (online supplementary table $2 \mathrm{~A}$ ) and four human patients (online supplementary table $2 \mathrm{~B}$ ). Mean $\Delta \Delta \mathrm{Ct}$ values were used to calculate expression fold changes compared with the region of minimal expression. (A) Mouse gene expression category 1 Wnt target genes (transit amplifying cells) showed quite variable expression along the intestine but were maximally expressed in the proximal small bowel (SB1) (with the exception of Cyclin D1). Category 2 genes (Paneth cells) were expressed in the small intestine as expected from this cell distribution $(\mathrm{p}<0.001$, analysis of variance (ANOVA)). Category 3 genes (stem-cell zone) showed very similar expression profiles with maximal expression in the small intestine declining markedly in the colon with minimal expression seen in the caecum ( $<<0.001$, ANOVA). Sox4 expression closely mirrored that of $A s c / 2$ and a striped line indicates the regions of identical expression. Conversely, epithelial Bmp2 expression was minimal in the duodenum climbing steadily to a peak in the mouse rectum $(p<0.001$, ANOVA), and there was also significant variability in the expression of some of the mesenchymal Wnt modulators (Grem2 and $\mathrm{Hgf}, \mathrm{p}<0.05$, ANOVA). The expression of the mesenchymal Wnt antagonist, Sfrp2, varied considerably with colonic expression more than 130 -fold greater than that in the small intestine ( $p=0.035$, ANOVA). (B) Human gene expression. All human Wnt target genes were maximally expressed in the ileum and minimally expressed in the rectum with the exception of the category 1 genes LEF1 and CYCLIN D1 (CCND1), which were maximally expressed more proximally. Category 3 (stem-cell) gene expression peaked in the ileum correlating with the human in situ findings $(p<0.001$, ANOVA). Caecal stem-cell marker expression is significantly greater than that seen in the rectum. The inverse correlation was seen for epithelial BMP2 expression ( $p<0.001$, ANOVA). Expression of the mesenchymal Wnt modulators was generally higher in the human small intestine which contrasted sharply with the murine Gremlin 1, 2 and Sfrp2 expression gradients. Only the expression gradient for HGF reached statistical significance in the humans ( $p=0.05$, ANOVA) as the result of a 10-fold difference in expression from the caecum to the rectum. SB1, proximal small bowel; SB2, mid-small bowel; SB3, distal small bowel. 
A

APC protein with major functional domains

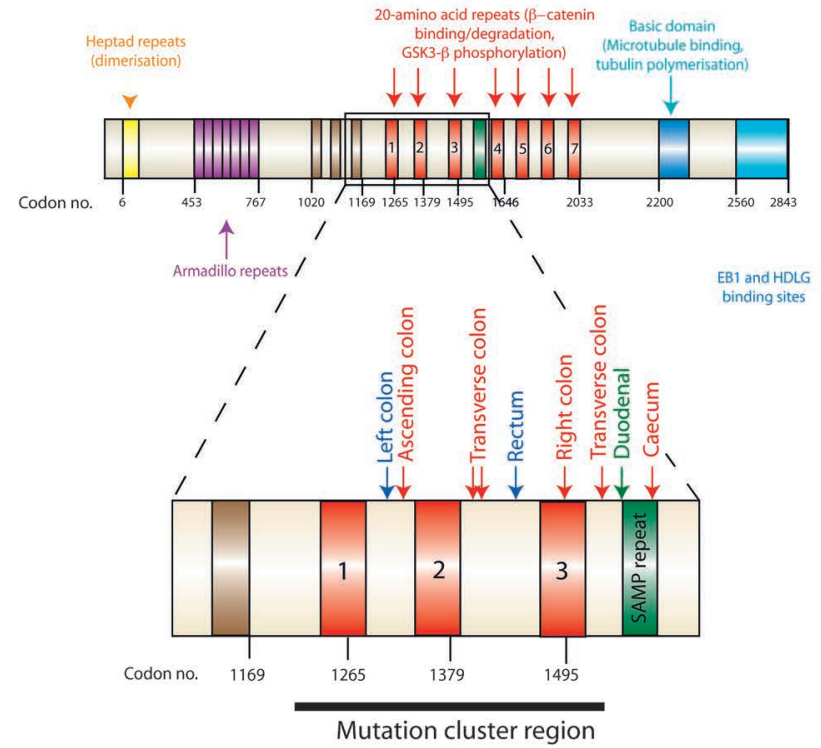

B Upper versus lower Gl in FAP

C Right versus left colon in sporadic CRC
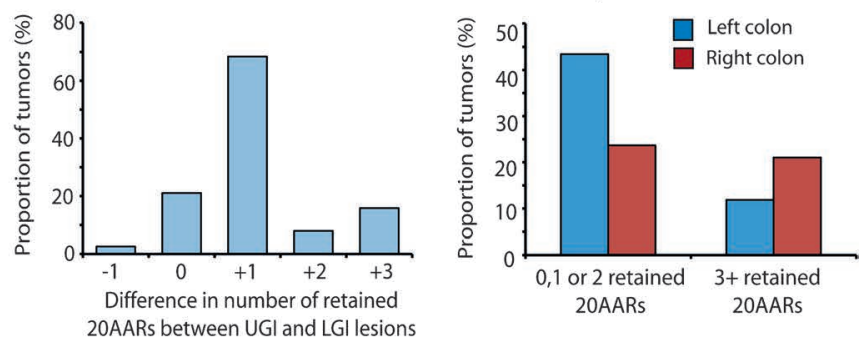

Figure 4 The adenomatous polyposis coli $(A P C)$ mutation spectrum varies with intestinal region in familial adenomatous polyposis (FAP) and sporadic gastrointestinal tumours. (A) Mutation spectra of upper and lower gastrointestinal (GI) lesions taken from the same FAP patient. The major functional domains of the APC protein are shown with an enlargement of the mutation cluster region. The number of retained 20 amino acid $\beta$-catenin binding repeats (20AARs) was calculated in upper $\mathrm{GI}$ (UGI) and lower GI (LGI) lesions taken from the same individual FAP patient(s) after determination of the mutation spectra of each lesion. The mutations for one individual patient (patient 1, online supplementary table 3) are mapped out (UGI lesion, green; right colon, red; left colon, blue) (B). UGI lesions in FAP retain significantly more 20AARs than LGI lesions taken from the same patient. The number of retained repeats from LGI lesions was subtracted from the number of repeats retained in the same patients' UGI lesions and the difference plotted. Significantly more repeats were retained in UGI lesions resulting in a more modest Wnt perturbation in these lesions $(p<0.001$, binomial test). (C) Right sided sporadic colorectal cancers and cell lines retain significantly more 20AARs than left sided lesions. Leisons in our sporadic data set of 76 tumours (online supplementary table $4 \mathrm{~A}$ and $\mathrm{B}$ ) were categorised by their distribution and the cumulative number of retained 20AARs after identification of first and second hits at $A P C$ in all lesions. Tumours retaining a cumulative total of three or more 20AARs were more likely to arise proximally whereas on the left side of the colon, mutations that retain fewer 20AARs were optimally selected to produce a greater Wnt perturbation in the resultant polyp ( $p=0.03$, Fishers Exact test).

\section{Stem-cell marker and Wnt target gene expression gradients are present in normal mouse intestine}

Our mouse model showed considerable phenotypic variation along the intestinal tract in response to pan-intestinal Wnt perturbation. We hypothesised that this might, in part, reflect underlying physiological gradients and so we also investigated the cephalo-caudal stem-cell and Wnt signalling gradients in the GI tract of wild-type litter-mates.

First, we used high-resolution ${ }^{3} \mathrm{H}$-UTP in situ assessment of mRNA for the stem-cell marker Lgr5 on well-orientated crypts (figure 2A) to allow accurate counting of the number of cells expressing stem-cell markers in the different intestinal regions. Lgr5 ISH showed a gradually decreasing stem-cell number progressing from $\mathrm{SB} 1$ to $\mathrm{SB} 3$, but then a marked reduction in the large bowel (ANOVA $\mathrm{p} \leq 0.001$ ) (figure $2 \mathrm{~B}$ ).

Next, we identified robust Wnt target genes based on consistent changes in a combination of experiments (details not shown): lithium chloride inhibition of GSK3 $\beta^{21}$ in the normal RIE-1 intestinal cell line (Leedham, unpublished data, 2010) and exon mRNA expression microarray and qRT-PCR in tumours from Apc-mutant mice. ${ }^{13}$ Wnt target genes were chosen and subdivided into the categories defined by Van der Flier et al: ${ }^{22}$

1. genes expressed in the rapidly dividing transit amplifying cells (Axin2, EphB2, CyclinD1, Lef1)

2. genes expressed by post-mitotic Paneth cells (EphB3, Mmp7 (mouse only))

3. genes expressed in the stem-cell position of the crypt (Lgr5, Olfm4 and Ascl2 (mouse only), Sox4 (mouse only)).

We then used qRT-PCR assays of identified Wnt target gene mRNA in order to measure overall expression levels in individual crypts taken from different regions of the mouse intestine (figure $3 \mathrm{~A}$ and online supplementary table 2A). Wnt target gene expression varied considerably down the length of the intestinal tract (figure 3A), with expression in the small bowel greater than that in the colon, correlating with the Lgr5 ISH findings. Minimal Wnt target expression was seen in the caecum.

We also demonstrated variability in the regional expression of some established epithelial and mesenchymal Wnt modulators (figure $3 \mathrm{~A}$ and online supplementary table 2A). There was a considerable gradient in expression of the Wnt antagonist Sfrp2 in the mouse, with mesenchymal expression in the colon more than 130-fold greater than that in the small intestine. Sfrp2 is a potent inhibitor of endogenous Wnt activity in rat normal intestinal epithelial cells (online supplementary results, online supplementary figure 2), and so its high expression is likely to contribute to the low epithelial Wnt activity in the mouse large bowel. Dickkopf 1, 2, 3 and $R$-spondin 1, 2, 3 expression were also measured but no appreciable intestinal gradient detected (data not shown).

\section{Human tumour mutation spectra and wnt gradient in the normal human intestines}

In $C t_{n n b} 1^{\Delta e x 3}$ mice, the distribution of dysplasia parallelled the physiological Wnt and stem-cell gradient. $\beta$-Catenin mutations are, however, rare in human bowel tumours which usually dysregulate Wnt through APC mutations. These mutations are under selective constraints such that stem cells acquire an optimal but not excessive level of Wnt signalling. 8 Existing data from different FAP patients support the existence of different $A P C$ mutation spectra in tumours from different locations in the bowel. ${ }^{10} 12{ }^{23}$ In order to compare the somatic mutation profiles of upper and lower GI tumours from the same patient, we studied a new set of FAP patients from whom gastric/duodenal and colonic lesions had been sampled. This allowed us to control for the effects of variation among patients and potential confounding factors such as diet. We demonstrated that upper GI lesions in FAP retain significantly more 20AARs than lower GI lesions taken from the same patient (figure 4 and online supplementary table 3). 


\section{A Mouse}

\section{i. Normal}

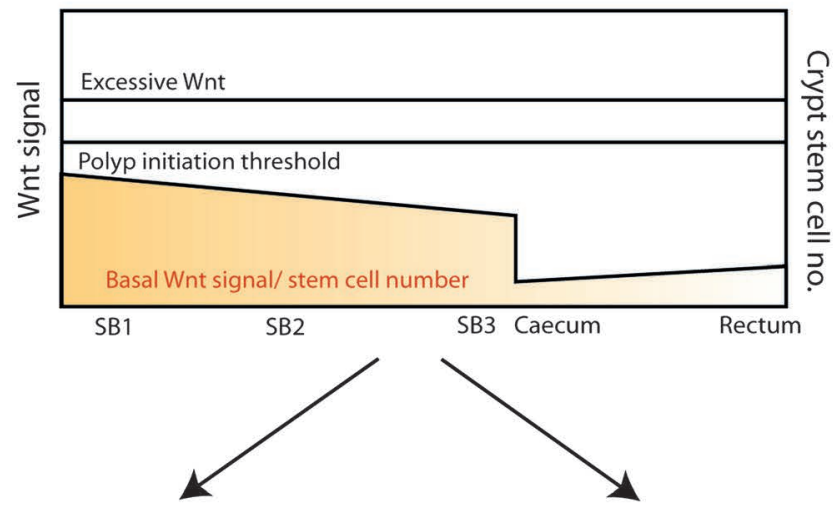

ii. Modest pathological Wnt

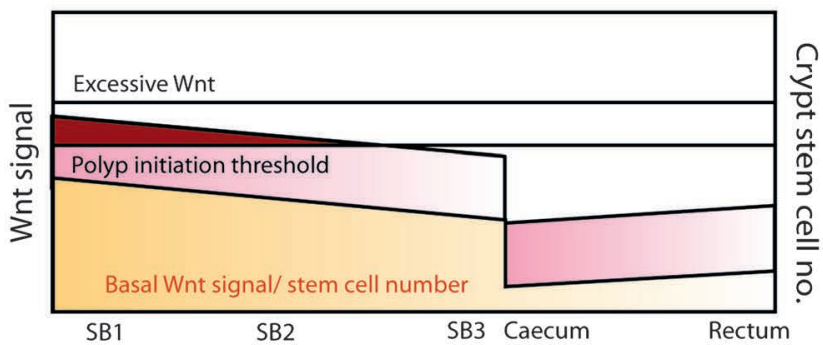

iii High pathological Wnt

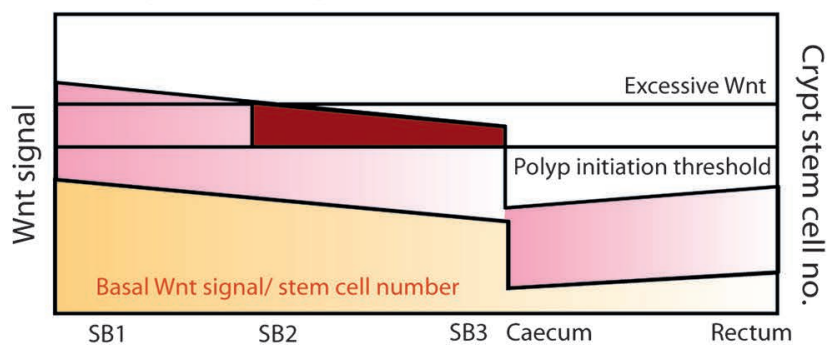

\section{B Human}

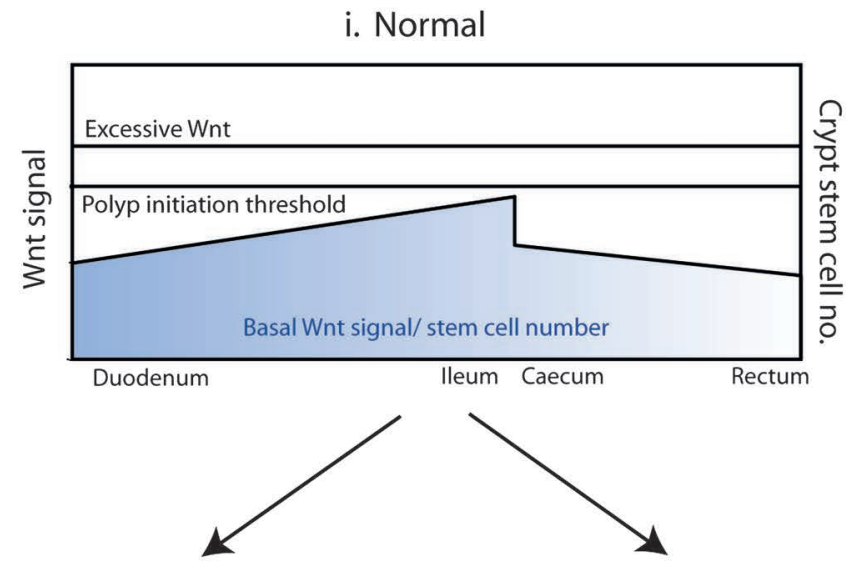

\section{ii. Modest pathological Wnt}

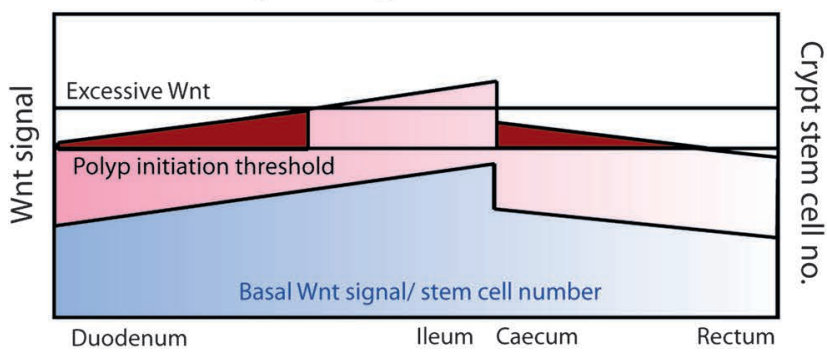

iii High pathological Wnt

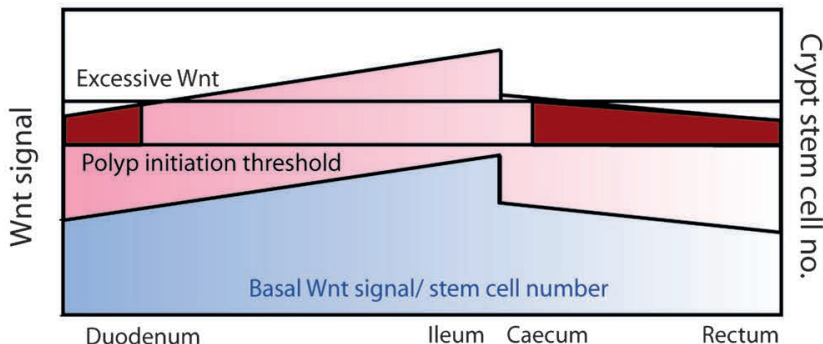

Figure 5 'Just-right' theory and genotype-phenotype correlations. (Ai and Bi) Basal Wnt signalling and stem-cell number in the mouse and humans. Mouse and human basal Wnt target expression profile and stem-cell number vary down the length of intestinal tract (figure 3). The two species have quite distinct profiles which in combination with 'just-right' mutation spectra may contribute to intestinal lesion distribution. (Aii and Aiii) The consequences of pathological Wnt signalling in the mouse. Submaximal pathological Wnt perturbation (seen in Ctnnb1 $1^{\Delta e \times 3}$ and $A p c^{1322 T}$ mice, as evidenced by low levels of nuclear $\beta$-catenin expression ${ }^{14}$ ) breaches the dysplasia threshold in the proximal small intestine where basal Wnt expression and stem-cell numbers are the highest, resulting in a heavy proximal small bowel (SB1) and mid-small bowel (SB2) lesion burden. In the mouse colon, the Wnt perturbation is insufficient to cause dysplasia in the colon despite causing an increase in the stem-cell number. Maximal Wnt perturbation as seen in the $A p c^{\operatorname{Min}(R 850 X)}$ is excessive and thus suboptimal proximally; hence, a predominantly distal small bowel (SB3) polyp distribution is characteristic. Maximal lesion distribution shown with dark red shading. (Bii and Biii) The consequences of pathological Wnt signalling in the humans. Modest Wnt perturbations such as that resulting from mutations that retain several 20 -amino acid $\beta$-catenin binding repeats (20AARs) 
Next, we genotyped and examined the mutation spectra of 19 sporadic colorectal cancers with known colonic distribution. This was combined with the data from 57 other sporadic tumours and cell lines taken from COSMIC v53. We only included lesions where we could identify both somatic hits at $A P C$ to give an accurate measure of the number of retained 20AARs (online supplementary table 4A,B). Right-sided lesions (transverse, ascending colon and caecum) had a higher frequency of mutations that retained a total of three or more 20AARs than left-sided colonic lesions (descending colon, sigmoid and rectum) ( $\chi^{2}$ test, $\mathrm{p}=0.03$ ). These data validate and add to the work by Albuquerque et $a l^{12}$ demonstrating that the optimal tumourigenic level of Wnt signal varies within the different regions of the colon in sporadic tumours as well as between the colon and small intestine in FAP (figure 4, online supplementary tables 3 and 4).

To our knowledge, physiological stem-cell and Wnt gradients have not previously been investigated in the normal human intestine. Based on our findings in the mouse we hypothesised that such gradients might contribute to established human tumour selective constraints. First, we assessed LGR5 mRNA expression in normal intestinal biopsy tissue taken from the duodenum, ileum and colon of the same patients. In contrast to the mouse, maximal stem-cell marker mRNA expression was seen in the ileum in humans (figure 2C).

Next, human Wnt target genes were chosen and categorised as for the mouse and their expression levels assayed in isolated crypts by qRT-PCR. Similar to the mouse, normal human Wnt target gene expression showed considerable variability down the length of the intestinal tract (figure $3 \mathrm{~B}$ and online supplementary table $2 \mathrm{~B}$ ). However, the majority of genes were maximally expressed in the ileum, consistent with the human LGR5 ISH results. Epithelial $B M P 2$ and mesenchymal $B M P$ antagonist expression was consistent with the observed stem-cell gradient. Mesenchymal HGF expression varied 10-fold between the caecum and rectum. As this is a potent morphogen that promotes nuclear $\beta$-catenin translocation in human CRC cells, ${ }^{24}$ this gradient may contribute to the observed difference in epithelial Wnt target gene activity between the human caecum and rectum. Dickkoff 1, 2, 3 and $R$-spondin 1, 2, 3 expression was also measured but no appreciable intestinal gradient was detected (data not shown).

\section{DISCUSSION}

Intestinal cell fate is determined by morphogen concentration gradients, mediated by complex interactions between an array of signalling molecules, receptors and antagonists. The importance of the Wnt pathway in the control of cell proliferation and the maintenance of intestinal stem cells is well established. Here, we have demonstrated variable stem-cell number and physiological Wnt pathway activity in different regions of the normal murine and human intestinal tracts.

Using a mouse model, we have also shown a graduated response to $\beta$-catenin stabilisation. All regions of the intestines showed an expansion of two overlapping populations of cells: (1) stem cells, predominantly at the crypt base and (2) prolif- erating, dysplastic cells, usually at the mid-crypt zone. The most profound changes were in SB1, where the entire epithelium was disordered and filled with stem-like, proliferating cells. In the large bowel, this stem and proliferating cell expansion was coupled with an increase in the crypt size and rate of fission, but normal architecture was largely retained and there was no dysplasia. As Villin-Cre ${ }^{E R T 2}$ drives Cre-mediated recombination homogenously throughout the crypt-villus axis and along the intestines, ${ }^{18}$ these data suggest an inherently variable response to increased Wnt signalling. Kuhnert et al showed that the effect of systemically antagonising Wnt by Dickkopf-1 expression similarly varied down the length of the murine intestine with the most profound effect in SB1. We demonstrated that this intrinsic sensitivity to Wnt modulation along the caudalcephalic axis of the intestine mirrored the physiological gradient expression of the stem-cell markers Lgr5 and Olfm4. Recent work has shown that the stem-cell marker Lgr5 functions as a Wnt receptor component that mediates Wnt signal enhancement via soluble R-spondin protein. ${ }^{25}$ As a Wnt signalling enhancer and target gene, Lgr5 appears to be subject to positive auto-regulation corroborated by the marked expansion of the Lgr5 expressing cell population following $\beta$-catenin stabilisation here, and in response to the wnt agonist R-spondin. ${ }^{26}$ As physiological $R$-spondin expression was consistent throughout the intestine we hypothesise that an intestinal gradient of Lgr5 expression may influence this regional inherent sensitivity to Wnt signalling and the response to pathological Wnt excess.

An intriguing question is whether the differences in dysplasia along the intestines of the $C_{n n b 1} 1^{\Delta e \times 3}$ mouse are related to our previous observations ${ }^{7}$ - confirmed in this manuscript-that specific APC mutation spectra are optimally selected in different regions of the human bowel. These observations, dubbed the 'just-right' model, ${ }^{8}$ propose that a threshold of Wnt signalling needs to be reached for dysplastic lesions to form, but that the optimum Wnt level for tumourigenesis is submaximal. Our mouse and human data provide clues to suggest that one reason for different $A P C$ mutations in tumours along the GI tract is a different physiological level of Wnt and stem-cell number. Hence, for example, when a modest pathological Wnt perturbation is superimposed on a low level of physiological Wnt-as in the colon of Ctnnb1 ${ }^{\Delta e \times 3}$ mice-the resultant Wnt level is still too low to cause dysplasia (figure 5A). In humans, the caecum has higher Wnt levels than the rectum, consistent with the selection of mutant APC alleles that retain a greater number of 20AARs. In microsatellite unstable cancers, this selection is favoured by the presence of multiple mononucleotide and dinucleotide repeats between codons 1450 and 1560 of APC that are prone to slippage in mismatch repair deficiency. Along with Albuquerque et $a l^{12}{ }^{27}$ we speculate that it is this retention of 20AARs in mismatch repair deficient tumours that accounts for the right-sided colonic distribution of these tumour types.

Homeostatic Wnt signalling and stem-cell numbers in the normal intestine are subject to auto-regulatory and combinatorial control with a complex network of interacting mesenchymal and epithelial morphogenetic pathways. ${ }^{28}$ We have demonstrated intestinal gradients of some important Wnt and

\footnotetext{
[Continued]

favour lesion development in the upper gastrointestinal (GI) and right colonic regions, explaining the more severe upper GI phenotype seen in patients with germline mutations after codon 1400. Conversely, early mutation cluster region mutations retain fewer 20AARs resulting in a greater degree of Wnt perturbation. Excessive Wnt is suboptimal for upper Gl lesions but favours more distal lesions resulting in a severe colonic phenotype with loss of heterozygosity optimally selected in the rectum of patients with germline codon 1309 mutations. ${ }^{10}$ Maximal lesion distribution is shown with dark red shading.
} 
stem-cell modulators such as Bmp2, Gremlin 1, 2 and $H g f$ in the epithelium and mesenchyme of humans and mice. Variable mesenchymal-epithelial cross talk along the intestinal tract is likely to influence physiological epithelial wnt activity. In particular, the marked Sfrp2 expression gradient between the mouse colon and small intestine may contribute to the very low level of physiological epithelial Wnt activity seen in the murine colon, as we have shown that Sfrp2 is a potent and dosedependent antagonist of endogenous Wnt activity (online supplementary figure 2). As this gradient in the mouse is the reverse of that seen in the humans (online supplementary figure 2 ), we hypothesise that the antagonism of Wnt activity by factors such as Sfrp 2 in the mouse colon may contribute to the differential intestinal polyp distribution in humans and mice. Interestingly, van Veelen et al have recently shown that coactivating intestinal Wnt signalling by Apc deletion and by a mechanism mimicking receptor tyrosine kinase phosphorylation of $\beta$-catenin markedly shifted murine intestinal tumour distribution towards the colon. ${ }^{29}$ This suggests that the maintenance of low physiological Wnt activity in the mouse colon by ligand sequestering antagonists such as Sfrp 2 may be partly counteracted by pathological activation of Wnt via an alternative (Wnt ligand and Frizzled receptor independent) mechanism, resulting in sufficient Wnt activity to breach the colonic Wnt signalling threshold and initiate colonic tumour formation. Regional variation in tyrosine kinase receptor expression has been reported in the normal human colon with epidermal growth factor receptor expression significantly higher proximally, ${ }^{30}$ correlating with the higher physiological Wnt activity we have demonstrated in the human right colon. Furthermore, variation in the pattern of epigenetic change has also been reported in the normal human large intestine. ${ }^{31}$ Methylation and transcriptional silencing of Wnt signalling genes have been shown to occur in morphologically normal crypts, ${ }^{32}$ and are common in colorectal tumours. ${ }^{33}$ Physiological regional variation in epigenetic transcriptional silencing or non-canonical Wnt activation may contribute to the marked variability in Wnt target gene activity demonstrated here.

We have generated a simple model in an attempt to explain multiple genetic events and complex physiological pathways and recognise the limitations of attempting to do this. There are a number of phenomena that this model cannot explain such as the disparity in prevalence between sporadic duodenal and caecal lesions in humans despite similar basal Wnt and stem-cell numbers in these two regions. While these data support the influence of Wnt signalling and stem-cell number variation on tumour distribution, there are also a number of potential confounding factors that have not been assessed such as the effect of intraluminal toxins and the contribution of the intestinal flora.

We propose that variable gradients in physiological Wnt activity and stem-cell number influence Wnt responsiveness along the crypt-villus and caudal-cephalic axes of the intestinal tract and that this influences regional intestinal mutation spectra and tumourigenic susceptibility. Identifying the homeostatic factors and Wnt modulators responsible for maintaining these gradients is likely to remain an area of research interest as the potential for therapeutic manipulation of Wnt in the colorectum remains a tantalising prospect.

Acknowledgements The authors thank the Animal technician staff at Cancer Research UK Clare Hall facility.

Funding This work was supported by Cancer Research UK (Clinician Scientist Fellowship to SJL) and the Wellcome Trust (CORE GRANT 090532/Z/09/Z).

\section{Competing interests None.}

Ethics approval All mouse work was approved by the UK Home Office and local ethics committee. Human archival tissue was obtained from St Marks Hospital, Harrow with multicentre ethics approval (MREC05/01606/66). Endoscopic biopsy tissue was obtained from the John Radcliffe Hospital, Oxford with local REC approval (REC 10/H0604/72).

Contributors Data acquisition by SJL, PRC, KH, AL, SS, HD, RJ and TAG. Pathology and material support by SM, MRJ, SK, SPLT, JE and SC. Study design and paper authorship by SJL and IPMT.

Provenance and peer review Not commissioned; internally peer reviewed.

Data sharing statement Unpublished data on lithium chloride stimulation of RIE cells and Dickkopf and $R$-Spondin Wnt modulator expression are available on request to the corresponding author.

Open access This is an open access article distributed under the terms of the Creative Commons Attribution Non-commercial, Non-Derivatives License, which permits use, distribution, and reproduction as is in any medium, provided the original work is properly cited, the work is not altered, the use is non-commercial and is otherwise in compliance with the license. See: http://creativecommons.org/licenses/ by-nc-nd/3.0/ and http://creativecommons.org/licenses/by-nc-nd/3.0/legalcode.

\section{REFERENCES}

1. Scoville D, Sato T, He X, et al. Current view: intestinal stem cells and signaling. Gastroenterology 2008;134:849-64.

2. Kuhnert F, Davis CR, Wang HT, et al. Essential requirement for Wnt signaling in proliferation of adult small intestine and colon revealed by adenoviral expression of Dickkopf-1. Proc Natl Acad Sci U S A 2004:101:266-71.

3. Radtke F, Clevers $\mathrm{H}$, Riccio 0 . From gut homeostasis to cancer. Curr Mol Med 2006:6:275-89.

4. Sieber $\mathbf{O M}$, Tomlinson IP, Lamlum $H$. The adenomatous polyposis coli (APC) tumour suppressor-genetics, function and disease. Mol Med Today 2000; 6:462-9.

5. Powell SM, Zilz N, Beazer-Barclay Y, et al. APC mutations occur early during colorectal tumorigenesis. Nature 1992;359:235-7.

6. Miyoshi Y, Nagase $\mathrm{H}$, Ando $\mathrm{H}$, et al. Somatic mutations of the APC gene in colorectal tumors: mutation cluster region in the APC gene. Hum Mol Genet 1992;1:229-33.

7. Lamlum $\mathbf{H}$, llyas $\mathrm{M}$, Rowan $\mathrm{A}$, et al. The type of somatic mutation at APC in familial adenomatous polyposis is determined by the site of the germline mutation: a new facet to Knudson's 'two-hit' hypothesis. Nat Med 1999;5:1071-5.

8. Albuquerque $\mathbf{C}$, Breukel $\mathrm{C}$, van der Luijt $\mathrm{R}$, et al. The 'just-right' signaling model: APC somatic mutations are selected based on a specific level of activation of the beta-catenin signaling cascade. Hum Mol Genet 2002;11:1549-60.

9. Nagase H, Miyoshi $Y$, Horii A, et al. Correlation between the location of germ-line mutations in the APC gene and the number of colorectal polyps in familia adenomatous polyposis patients. Cancer Res 1992:52:4055-7.

10. Will 0, Leedham S, Elia G, et al. Location in the large bowel influences the APC mutations observed in FAP adenomas. Fam Cancer 2010;9:389-93.

11. Groves CJ, Saunders BP. Spigelman AD, et al. Duodenal cancer in patients with familial adenomatous polyposis (FAP): results of a 10 year prospective study. Gut 2002; 50:636-41.

12. Albuquerque $\mathbf{C}$, Baltazar $\mathrm{C}$, Filipe $\mathrm{B}$, et al. Colorectal cancers show distinct mutation spectra in members of the canonical WNT signaling pathway according to their anatomical location and type of genetic instability. Genes Chromosomes Cancer 2010;49:746-59

13. Lewis A, Segditsas S, Deheragoda M, et al. Severe polyposis in Apc(1322T) mice is associated with submaximal Wnt signalling and increased expression of the stem cell marker Lgr5. Gut 2010;59:1680-6.

14. Pollard P, Deheragoda M, Segditsas S, et al. The Apc 1322T mouse develops severe polyposis associated with submaximal nuclear beta-catenin expression. Gastroenterology 2009;136:2204-13.e1-13

15. Sansom $\mathbf{0 J}$, Reed KR, Hayes AJ, et al. Loss of Apc in vivo immediately perturbs Wnt signaling, differentiation, and migration. Genes Dev 2004;18:1385-90.

16. Fodde R, Kuipers J, Rosenberg C, et al. Mutations in the APC tumour suppressor gene cause chromosomal instability. Nat Cell Biol 2001;3:433-8.

17. Harada N, Tamai Y, Ishikawa $T$, et al. Intestinal polyposis in mice with a dominant stable mutation of the beta-catenin gene. EMBO J 1999;18:5931-42.

18. El Marjou F, Janssen $\mathrm{K}$, Chang B, et al. Tissue-specific and inducible Cre-mediated recombination in the gut epithelium. Genesis 2004;39:186-93

19. Thirlwell C, Will O, Domingo E, et al. Clonality assessment and clonal ordering of individual neoplastic crypts shows polyclonality of colorectal adenomas. Gastroenterology 2010;138:1441-54.e1-7.

20. Barker N, van Es J, Kuipers J, et al. Identification of stem cells in small intestine and colon by marker gene Lgr5. Nature 2007:449:1003-7.

21. Stambolic V, Ruel L, Woodgett JR. Lithium inhibits glycogen synthase kinase-3 activity and mimics wingless signalling in intact cells. Curr Biol 1996:6:1664-8.

22. Van der Flier LG, Sabates-Bellver J, Oving I, et al. The intestinal Wnt/TCF signature. Gastroenterology 2007;132:628-32. 
23. Groves C, Lamlum H, Crabtree $\mathrm{M}$, et al. Mutation cluster region, association between germline and somatic mutations and genotype-phenotype correlation in upper gastrointestinal familial adenomatous polyposis. Am J Pathol 2002:160:2055-61.

24. Vermeulen L, De Sousa EMF, van der Heijden M, et al. Wnt activity defines colon cancer stem cells and is regulated by the microenvironment. Nat Cell Biol 2010;12:468-76.

25. de Lau W, Barker N, Low TY, et al. Lgr5 homologues associate with Wnt receptors and mediate R-spondin signalling. Nature 2011;476:293-7.

26. Ootani A, Li X, Sangiorgi E, et al. Sustained in vitro intestinal epithelial culture within a Wnt-dependent stem cell niche. Nat Med 2009;15:701-6.

27. Albuquerque C, Bakker ER, van Veelen W, et al. Colorectal cancers choosing sides. Biochim Biophys Acta 2011;1816:219-31.

28. Crosnier C, Stamataki D, Lewis J. Organizing cell renewal in the intestine: stem cells, signals and combinatorial control. Nat Rev Genet 2006;7:349-59.
29. van Veelen W, Le NH, Helvensteijn W, et al. $\beta$-catenin tyrosine 654 phosphorylation increases Wnt signalling and intestinal tumorigenesis. Gut 2011;60:1204-12.

30. Koenders PG, Peters WH, Wobbes T, et al. Epidermal growth factor receptor levels are lower in carcinomatous than in normal colorectal tissue. Br J Cancer 1992;65:189-92.

31. Figueiredo JC, Grau MV, Wallace K, et al. Global DNA hypomethylation (LINE-1) in the normal colon and lifestyle characteristics and dietary and genetic factors. Cancer Epidemiol Biomarkers Prev 2009;18:1041-9.

32. Belshaw NJ, Pal N, Tapp HS, et al. Patterns of DNA methylation in individual colonic crypts reveal aging and cancer-related field defects in the morphologically normal mucosa. Carcinogenesis 2010;31:1158-63.

33. Segditsas S, Sieber OM, Rowan A, et al. Promoter hypermethylation leads to decreased APC mRNA expression in familial polyposis and sporadic colorectal tumours, but does not substitute for truncating mutations. Exp Mol Pathol 2008:85:201-6. 